\title{
SINS/GPS Carrier Phase Rate Integrated Navigation System based on Square-root CKF
}

\author{
http://dx.doi.org/10.3991/ijoe.v10i1.3166 \\ Lin Zhao, Zhenguo Yuan \\ College of Automation, Harbin Engineering University, Haerbin, China
}

\begin{abstract}
The integration of Strap-down Inertial Navigation System (SINS) and Global Positioning System(GPS)can not merely reduce the error of navigation system, which would enhance the system dynamic performance. GPS Carrier phase rate is a high-precision measurement without integer ambiguity problem. Moreover, only one GPS receiver is needed for SINS/GPS carrier phase rate integrated navigation system. The measurement of GPS carrier phase rate is derived and a integrated scheme of SINS and GPS carrier phase rate is proposed. Common used nonlinear filter, extended Kalman filter (EKF) has high-order truncation error which affects the system accuracy. An improved cubature Kalman filter (CKF) used square root is introduced. The square-root CKF calculation avoids calculating the Jacobian matrix and has better estimate precision.
\end{abstract}

Index Terms-SINS, Carrier phase rate, integration, CKF, Square-root

\section{INTRODUCTION}

GPS carrier phase rate eliminates the integer ambiguity problem. The velocity of the carrier can be obtained by deriving the GPS carrier rate, which can be used to combine SINS[1] to be a loose coupled system[2].As a original Observation, carrier phase rate can also be combined with output from SINS[3]. The carrier phase rate measurement model is derived and the combination principle is explanted.

To the nonlinear model, EKF is effective only when the nonlinear system is approximate linear, which reach only first order accuracy[4].And EKF needs to calculate the Jacobian matrix, which is trivial. In order to improve the precision of the intergraded system, square-root CKF is introduced to make nonlinear filtering. According to spherical-radial cubature rule[5], CKF does't need to linearize the nonlinear equation and it can reach three order accuracy[6].Commonly used Kalman filter has stability problem. The square-root CKF can ensure half positive definitiveness of the covariance matrix and improve the stability of filter process. Results show that square-root $\mathrm{CKF}$ can get less error than EKF for the integrated system.

\section{MEASUREMENT OF CARRIER PHASE RATE}

The equation of GPS carrier phase is[7]:

$$
\varphi=R-I+T+c\left(\delta t_{r}-\delta t_{s}\right)+O+M+\lambda N
$$

Where, $\varphi$ is carrier phase, $R$ is the distance from receiver to the satellite, $I$ is ionospheric error, $T$ is troposphere error, $\delta t_{s}$ is satellite clock error, $\delta t_{u}$ is receiver clock error, $O$ is satellite orbit error, $M$ is the measurement error of multipath and receiver noise, $\lambda$ is wavelength and $N$ is integer ambiguity. To the same satellite, the carrier phase $\varphi\left(t_{1}\right)$ deducts carrier phase $\varphi\left(t_{2}\right)$. When time interval is small enough (less than one second), the change of some error can be eliminated or corrected by model correction and navigation message correction. $N$ is fixed constant that can also be eliminated by the difference. So the carrier phase rate is obtained:

$$
\Delta \varphi=\varphi\left(t_{2}\right)-\varphi\left(t_{1}\right)=\Delta R+c \Delta \delta t_{r}
$$

The position relation of receiver and satellite at adjoining time is shown in figure 1 .

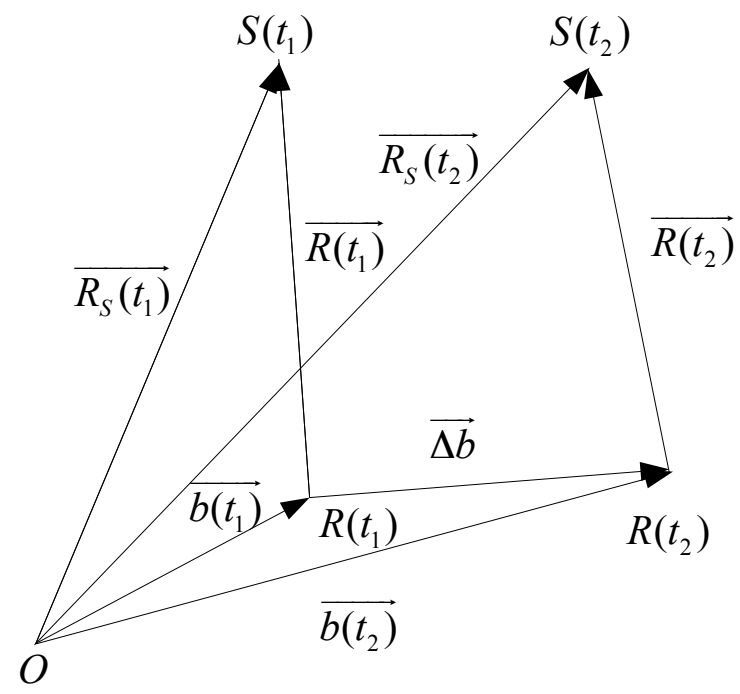

Figure 1. The position relation of receiver and satellite at adjoining time

Where, the position of receiver at different time is $R\left(t_{1}\right)$ and $R\left(t_{2}\right)$, the position of the satellite is $S\left(t_{1}\right)$ and $S\left(t_{2}\right), \overrightarrow{R_{S}\left(t_{i}\right)}, i=1,2$ represents the vector of satellite position, $\overrightarrow{b\left(t_{i}\right)}, i=1,2$ represents the vector of receiver position, $\overrightarrow{R\left(t_{i}\right)}, i=1,2$ represents the vector from satellite to receiver, $\overrightarrow{\Delta b}$ represents the change of the receiver position vector. According to the position relationship: 


$$
\overrightarrow{R\left(t_{1}\right)}=\left(\overrightarrow{R_{S}\left(t_{1}\right)}-\overrightarrow{b\left(t_{1}\right)}\right) \cdot \overrightarrow{e\left(t_{1}\right)}
$$

Where, $\overrightarrow{e\left(t_{1}\right)}\left|\overrightarrow{R_{S}\left(t_{1}\right)}-\overrightarrow{b\left(t_{1}\right)}\right|=\overrightarrow{R_{S}\left(t_{1}\right)}-\overrightarrow{b\left(t_{1}\right)}, \overrightarrow{e\left(t_{1}\right)}$ is unit vector at $t_{1}$ from receiver to the satellite.

$$
\begin{aligned}
& \overrightarrow{\Delta R}=\overrightarrow{R\left(t_{2}\right)}-\overrightarrow{R\left(t_{1}\right)}= \\
& \left(\overrightarrow{R_{S}\left(t_{2}\right)}-\overrightarrow{b\left(t_{2}\right)}\right) \cdot \overrightarrow{e\left(t_{2}\right)}- \\
& \left(\overrightarrow{R_{S}\left(t_{1}\right)}-\overrightarrow{b\left(t_{1}\right)}\right) \cdot \overrightarrow{e\left(t_{1}\right)}= \\
& \overrightarrow{R_{S}\left(t_{2}\right)} \cdot \overrightarrow{e\left(t_{2}\right)}-\overrightarrow{R_{S}\left(t_{1}\right)} \cdot \overrightarrow{e\left(t_{1}\right)} \\
& -\overrightarrow{b\left(t_{2}\right)} \cdot \overrightarrow{e\left(t_{2}\right)}+\overrightarrow{b\left(t_{1}\right)} \cdot \overrightarrow{e\left(t_{1}\right)}
\end{aligned}
$$

Due to $\overrightarrow{\Delta b}=\overrightarrow{b\left(t_{2}\right)}-\overrightarrow{b\left(t_{1}\right)}, \overrightarrow{b\left(t_{2}\right)}$ can be eliminated.

$$
\begin{gathered}
\overrightarrow{\Delta R}=\overrightarrow{R_{S}\left(t_{2}\right)} \cdot \overrightarrow{e\left(t_{2}\right)}-\overrightarrow{R_{S}\left(t_{1}\right)} \cdot \overrightarrow{e\left(t_{1}\right)} \\
-\overrightarrow{b\left(t_{1}\right)} \cdot \overrightarrow{e\left(t_{2}\right)}-\overrightarrow{\Delta b} \cdot \overrightarrow{e\left(t_{2}\right)}+\overrightarrow{b\left(t_{1}\right)} \cdot \overrightarrow{e\left(t_{1}\right)} \\
\overrightarrow{R_{S}\left(t_{2}\right)} \cdot \overrightarrow{e\left(t_{2}\right)}-\overrightarrow{R_{S}\left(t_{1}\right)} \cdot \overrightarrow{e\left(t_{1}\right)}=\text { Doppler represents }
\end{gathered}
$$

Doppler frequency shift.

$$
\overrightarrow{b\left(t_{1}\right)} \cdot \overrightarrow{e\left(t_{2}\right)}-\overrightarrow{b\left(t_{1}\right)} \cdot \overrightarrow{e\left(t_{1}\right)}=\text { Geometry represents }
$$

the geometric position change of the satellite. They can be obtained from navigation message and measurement of GPS. So the carrier phase rate measurement is:

$$
\begin{aligned}
\Delta \varphi= & -\overrightarrow{\Delta b} \cdot \overrightarrow{e\left(t_{2}\right)}+\text { Doppler } \\
& - \text { Geometry }+\mathrm{c} \Delta \delta t_{r}
\end{aligned}
$$

\section{MEASUREMENT OF SINS}

The error equation of SINS reflects the relationship between inertial components error and positioning error.

On the basis of literature, SINS error model is:

$$
\begin{aligned}
& \delta \dot{\boldsymbol{v}}=\left[\boldsymbol{I}-\left(\boldsymbol{C}_{t}^{t}\right)^{T}\right] \boldsymbol{C}_{b}^{t} \hat{\boldsymbol{f}}^{b}+\boldsymbol{C}_{b}^{t} \delta \boldsymbol{f}^{b}- \\
& \left(2 \hat{\boldsymbol{\omega}}_{i e}^{t}+\hat{\boldsymbol{\omega}}_{e t}^{t}\right) \times \delta \boldsymbol{v}-\left(2 \delta \boldsymbol{\omega}_{i e}^{t}+\delta \boldsymbol{\omega}_{e t}^{t}\right) \times \boldsymbol{v}+\delta \boldsymbol{g} \\
& \dot{\boldsymbol{\phi}}=\left(\boldsymbol{I}-\boldsymbol{C}_{t}^{t}\right) \hat{\boldsymbol{\omega}}_{i t}^{t}+\delta \boldsymbol{\omega}_{i t}^{t}-\boldsymbol{C}_{b}^{t} \delta \boldsymbol{\omega}_{i b}^{b}
\end{aligned}
$$

$\delta \boldsymbol{v}$ is velocity error vector, $\phi$ is misalignment angle vector, $\boldsymbol{C}_{b}^{t}$ is direction cosine matrix from carrier coordinate to geography coordinate, $\boldsymbol{C}_{b}^{t^{\prime}}$ is direction cosine matrix from carrier coordinate to calculated geography coordinate. $\hat{\boldsymbol{f}}^{b}$ is real proportional output, $\delta \boldsymbol{f}^{b}$ and $\delta \boldsymbol{\omega}_{i b}^{b}$ are measurement error, $\hat{\boldsymbol{\omega}}_{i e}^{t}$ is earth rotation angle rate in calculated geography coordinate, $\hat{\boldsymbol{\omega}}_{e t}^{t}$ is rotation angle rate relative to geography coordinate, $\hat{\boldsymbol{\omega}}_{i t}^{t}$ is rotation angle rate relative to inertial coordinate, $\delta \boldsymbol{g}$ is error of gravity acceleration.
$\left[\begin{array}{lll}L & \lambda & h\end{array}\right]$ is the position, $\left[\begin{array}{lll}v_{E} & v_{N} & v_{U}\end{array}\right]$ is the vielocity, the position error is:

$$
\begin{aligned}
& \delta \dot{L}= \frac{1}{R_{M}+h} \delta v_{E}-\frac{v_{N}}{\left(R_{M}+h\right)^{2}} \delta h \\
& \delta \dot{\lambda}= \frac{1}{\left(R_{N}+h\right) \cos L} \delta v_{E}-\frac{1}{\left(R_{N}+h\right)^{2} \cos L} \delta h \\
& \quad+\frac{\tan L}{\left(R_{N}+h\right)^{2} \cos L} \delta L \\
& \delta \dot{h}=\delta v_{U}
\end{aligned}
$$

\section{THE INTEGRATION OF SINS AND CARRIER PHASE}

The position can be obtained from SINS. According to the GPS time interval, the change of the receiver position $\Delta b$ can be predicted. The navigation information like almanac data from GPS receiver can be transferred to SINS. The carrier phase rate can be obtained. The scheme is shown in figure 2 :

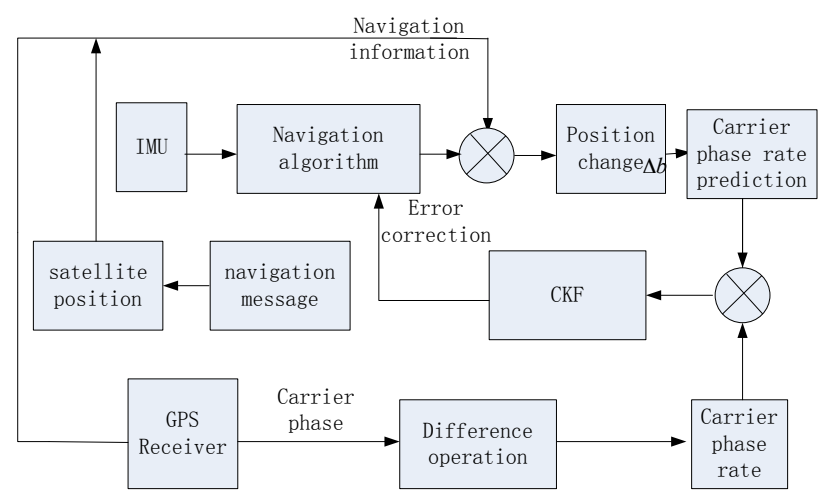

Figure 2. The position relationship of receiver and satellite

The velocity calculated from SINS make integration in 1 second time interval, which can obtain position increment in 1 second time interval. The prediction of carrier phase rate can be obtained by projecting this position increment to the line of satellite sight. Square-root CKF is used to make data fusion and make calibration and compensation of SINS.

\section{SQUARE-ROOT CKF}

Square-root CKF is based on Cubature rule. The state space form of discrete nonlinear System is[8]:

$f\left(x_{k-1}\right)$ and $h\left(x_{k}\right)$ are known arbitrary function.

$$
\left\{\begin{array}{l}
x_{k}=f\left(x_{k-1}\right)+w_{k-1} \\
z_{k}=h\left(x_{k}\right)+v_{k}
\end{array}\right.
$$

$w_{k-1} \sim N(0, Q)$ is random system noise, $v_{k} \sim N(0, R)$ is observation noise.

In order to deal with the filtering problem with Gauss distribution, use the general form of filter[9]: 


$$
\begin{aligned}
& x_{k \mid k}=\hat{x}_{k \mid k-1}+S_{k}\left(z_{k}-\hat{z}_{k}\right) \\
& P_{k \mid k}=P_{k \mid k-1}-S_{k} P_{z z} S_{k}^{T} \\
& S_{k}=P_{x z} P_{z z}^{-1}
\end{aligned}
$$

Where, the variable above is calculated:

$\hat{x}_{k \mid k-1}=E\left[f\left(x_{k-1}\right)\right]$

$=\int_{R^{n}} f\left(x_{k-1}\right) p\left(x_{k-1}\right) d x_{k-1}$

$=\int_{R^{n}} f\left(x_{k-1}\right) \times N\left(x_{k-1} ; P_{k-1 \mid k-1}\right) d x_{k-1}$

$P_{k \mid k-1}=E\left[\left(x_{k}-\hat{x}_{k \mid k-1}\right)\left(x_{k}-\hat{x}_{k \mid k-1}\right)^{T}\right]$

$=-\hat{x}_{k \mid k-1} \hat{x}_{k \mid k-1}^{T}+\int_{R^{n}} f\left(x_{k-1}\right) f^{T}\left(x_{k-1}\right) \times$,

$N\left(x_{k-1} ; P_{k-1 \mid k-1}\right) d x_{k-1}+Q_{k-1}$

$$
\hat{z}_{x}=E\left[h\left(x_{k}\right)\right]
$$

$=\int_{R^{n}} h\left(x_{k}\right) \times N\left(x_{k} ; P_{k \mid k-1}\right) d x_{k}$,

$P_{z z}=E\left[\left(z_{k}-\hat{z}_{x}\right)\left(z_{k}-\hat{z}_{x}\right)^{T}\right]$

$=\int_{R^{n}} h\left(x_{k}\right) h^{T}\left(x_{k}\right) \times N\left(x_{k} ; P_{k \mid k-1}\right) d x_{k}$,

$-\hat{z}_{k \mid k-1} \hat{z}_{k \mid k-1}^{T}+R_{k}$

$P_{x z}=E\left[\left(x_{k}-\hat{x}_{x}\right)\left(z_{k}-\hat{z}_{x}\right)^{T}\right]$

$=\int_{R^{n}} x_{k} h^{T}\left(x_{k}\right) \times N\left(x_{k} ; P_{k \mid k-1}\right) d x_{k}$.

$-\hat{x}_{k \mid k-1} \hat{z}_{k \mid k-1}^{T}$

The calculation of mathematical expectation above involves integration with same dimension:

$$
I(f)=\int_{R^{n}} f(x) \exp \left(-x^{T} x\right) d x
$$

Where, $f(g)$ is arbitrary function, $R^{n}$ is integral region.The integration can be calculated by Spherical-radial cubature rule[7], which is not the thesis focused of this paper. The step of square-root is as follows:

(1) Time update

The square-rooting matrix of error covariance at time $k-1$ is $S(k-1 \mid k-1)$, the optimal estimation is $\hat{x}(k-1 \mid k-1)$. So the volume point is :

$$
\begin{aligned}
& x_{i}(k \mid k-1)=S(k \mid k-1) \xi_{i}+x(k \mid k-1) \\
& \xi_{i}=\left\{\begin{array}{c}
\sqrt{m / 2} \cdot \varepsilon_{i}, i=1,2 \mathrm{~L} n . \\
\sqrt{m / 2} \cdot \varepsilon_{i-n}, i=n+1, n+2 \mathrm{~L} 2 n .
\end{array},\right.
\end{aligned}
$$

the dimension of state vector.

The transmission of the volume point is:

$$
\tilde{x}_{i}(k \mid k-1)=f\left(x_{i}(k-1 \mid k-1)\right)
$$

The estimation of state vector is:

$$
\hat{x}(k \mid k-1)=\frac{1}{m} \sum_{i=1}^{m} \tilde{x}_{i}(k \mid k-1)
$$

The square root of error covariance is:

$$
S(k \mid k-1)=\operatorname{tri}\left[\tilde{X}(k \mid k-1) S_{Q}(k)\right]
$$

Where, tri means triangle operation, $S_{Q}$ is the square root of process noise covariance (QR decomposition method is used here).

Where,

$$
\begin{array}{r}
\tilde{X}(k \mid k)=\frac{1}{\sqrt{m}}\left[\tilde{X}_{1}(k \mid k-1)-\hat{x}(k \mid k-1)\right], \\
\tilde{X}_{2}(k \mid k-1)-\hat{x}(k \mid k-1), \\
\vdots \\
\tilde{X}_{m}[(k \mid k-1)-\hat{x}(k \mid k-1)]
\end{array}
$$

(2) Measurement update

Update Volume point calculating is:

$$
\begin{gathered}
x_{i}(k \mid k-1)=S(k \mid k-1) \zeta_{i}+x(k \mid k-1) \\
z_{i}(k \mid k-1)=M(k) x_{i}(k \mid k-1)
\end{gathered}
$$

Measurement forecast is:

$$
\hat{z}(k \mid k-1)=\frac{1}{m} \sum_{i=1}^{m} z_{i}(k \mid k-1)
$$

Square root of forecast covariance matrix is:

$$
S_{z z}(k \mid k-1)=\operatorname{tri}\left[z(k \mid k-1) S_{R}(k)\right]
$$

Where,

$$
\begin{array}{r}
z(k \mid k-1)=\frac{1}{\sqrt{m}}\left[z_{1}(k \mid k-1)-\hat{z}(k \mid k-1),\right] \\
z_{1}(k \mid k-1)-\hat{z}(k \mid k-1) \\
\mathrm{M} \\
z_{m}(k \mid k-1)-\hat{z}(k \mid k-1)
\end{array}
$$

and

$S_{R}$ is the square root of measurement noise covariance.

Cross-covariance matrix is:

$$
P_{x z}(k \mid k-1)=x(k \mid k-1) z^{T}(k \mid k-1)
$$

Where,

$$
\begin{array}{r}
x(k \mid k)=\frac{1}{\sqrt{m}}\left[x_{1}(k \mid k-1)-\hat{x}(k \mid k-1)\right] \\
x_{2}(k \mid k-1)-\hat{x}(k \mid k-1), \\
\mathrm{M} \\
x_{m}(k \mid k-1)-\hat{x}(k \mid k-1)
\end{array}
$$

Gain matrix is:

$$
\begin{aligned}
& K(k)=P_{x z}(k \mid k-1) \cdot \\
& {\left[S_{z z}^{T}(k \mid k-1)\right]^{-1} \times S_{z z}(k \mid k-1)^{-1}}
\end{aligned}
$$

State update is:

$$
\begin{aligned}
& \hat{x}(k \mid k)=\hat{x}(k \mid k-1)+ \\
& K(k)[z(k)-\hat{z}(k \mid k-1)]
\end{aligned}
$$

Square root of calculated covariance matrix is: 


$$
\begin{aligned}
& S(k \mid k)=\operatorname{tri}[x(k \mid k-1)- \\
& K(k) z(k)-\hat{z}(k \mid k-1)] \times K(k) S_{R}(k)
\end{aligned}
$$

\section{SIMULATION AND EXPERIMENTAL RESULTS}

Use HG1700 AG17 IMU and NOVATEL receiver platform, the vehicle reroutes the school for two cycles. Records the data and the vehicle curves is shown in figure3:

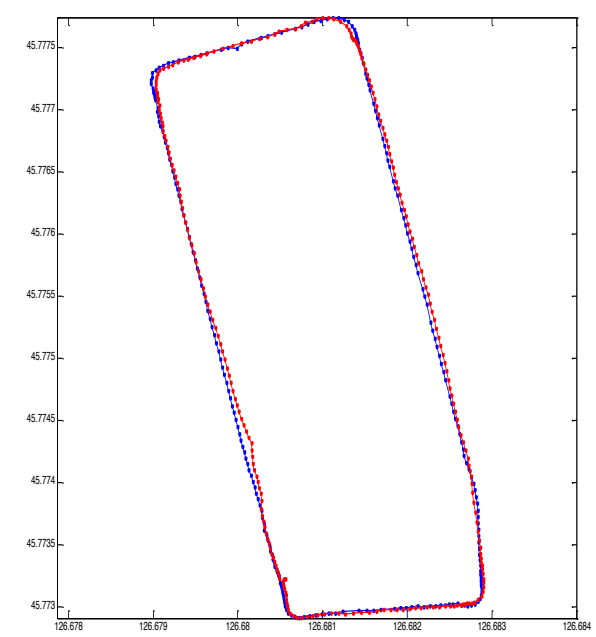

Figure 3. Vehicle curves

Lateral axis is latitude, longitudinal axis is longitude. Under the same condition, deal with data with square-root CKF and EKF, the result is shown in figure4-5:
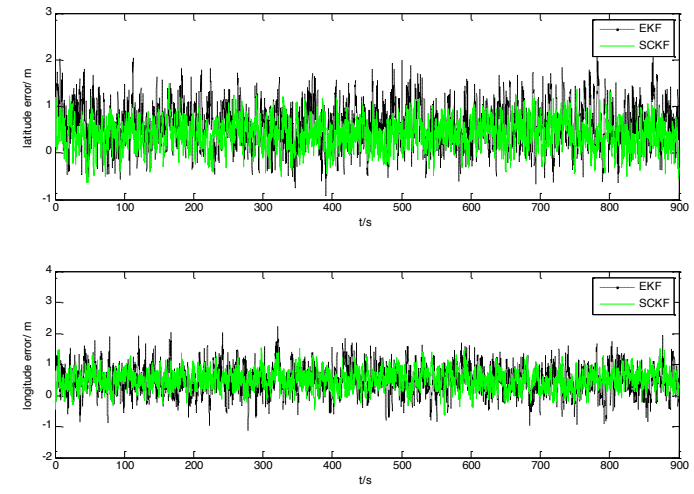

Figure 4. Position error
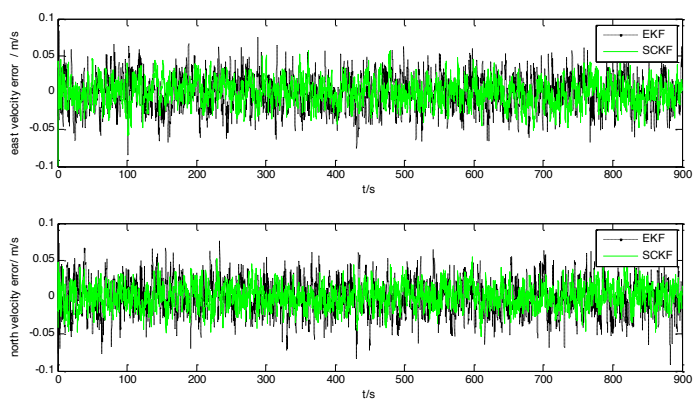

Figure 5. Velocity error
Using EKF, the error of position and velocity is $0.61 \mathrm{~m}, 0.55 \mathrm{~m}$ and $0.0473 \mathrm{~m} / \mathrm{s}$, while, using square-root $\mathrm{CKF}$, the error of position and velocity is $0.37 \mathrm{~m}, 0.35 \mathrm{~m}$ and $0.0319 \mathrm{~m} / \mathrm{s}$.

The integration of SINS and GPS carrier phase rate makes full use of high-precision carrier phase without integer ambiguity calculation. The improved Cubature Kalman filter is based on cubature rule and calculate the mean and covariance of nonlinear function directly. square-root $\mathrm{CKF}$ can also improve the estimate accuracy.

SCKF has no need to linearize the nonlinear model and does not depend on the specific model of nonlinear equations. Algorithm of SCKF is relatively independent and applicable to most form of non-linear model.

Simulation shows that square-root CKF can reduce the error of position and velocity better than EKF.

\section{REFERENCES}

[1] Frank van Grass,Andery Soloviev. Precise velocity estimation using a stand-alone GPS Receiver [C].ION NTM 2003, Anaheim, America, 2003.

[2] Bin Jia, Ming Xin and Yang Cheng. Sparse Gauss-Hermite Quadrature Filter with application to Spacecraft Attitude Estimation [J]. Journal of Guidance Control and Dynamics. 2011, 34(2):pp367-379. http://dx.doi.org/10.2514/1.52016

[3] Ben K. H. Soon, Steve Scheding,Hyung-Kuen Lee. An approach to aid INS using time-differenced GPS carrier phase (TDCP) measurements [J]. GPS Solut 2008, (12): 261-271. http://dx.doi.org/10.1007/s10291-007-0083-7

[4] K. Ito and K. Q. Xiong. Gaussian filters for nonlinear filtering problems [J]. IEEE Transactions on Automatic Control. 2000, 45(5): 910-927. http://dx.doi.org/10.1109/9.855552

[5] Wenling Li and Yingmin Jia. Location of Mobile Station with Maneuvers Using an IMM-Based Cubature Kalman Filter. IEEE Transactions on Industrial Electronics[C]. International Conference on Mechatronics and Automation August, Beijing, China, 2011.

[6] Chao Li and Quan-Bo Ge. SCKF for Mav Attitude Estimation [C]. Proceedings of the 2011 International Conference on Machine Learning and Cybernetics. 2011. http://dx.doi.org/10.1109/ICMLC.2011.6016854

[7] Feng Sun,Lijun Tang.Augmented and Non-augmented Cubature Kalman Filter[J]. Journal of Information and Computational Science.2012,9(2):437-450.

[8] H. Pesonen and R. Piche. Cubature-based Kalman filters for positioning[C]. Workshop on Positioning Navigation and Communication. 2010.

[9] K.Ito and K.Q.Xiong. Gaussian filters for non- linear filtering problem [J]. IEEE Transactions on Automatic Control, 2000, 45 (5): 910-927. http://dx.doi.org/10.1109/9.855552

\section{AUTHORS}

Lin Zhao is with the College of Automation, Harbin Engineering University, Haerbin, CO 150001 China.

Zhenguo Yuan is with the College of Automation, Harbin Engineering University, Haerbin, CO 150001 China (e-mail: yuanzhenguodaohang@163.com).

This research was supported by the National Natural Science Foundation of China Grant (61273081). Submitted, 06 September 2013. Published as resubmitted by the authors on 23 January 2014. 